\title{
On the Semi-Discrete Davey-Stewartson System with Self-Consistent Sources
}

\author{
Gegenhasi \\ School of Mathematical Science, Inner Mongolia University, Hohhot, China \\ Email: gegen@amss.ac.cn
}

Received 1 April 2015; accepted 5 May 2015; published 11 May 2015

Copyright (c) 2015 by author and Scientific Research Publishing Inc.

This work is licensed under the Creative Commons Attribution International License (CC BY).

http://creativecommons.org/licenses/by/4.0/

(c) (i) Open Access

\begin{abstract}
A differential-difference Davey-Stewartson system with self-consistent sources is constructed using the source generation procedure. We observe how the resulting coupled discrete system reduces to the identities for determinant by presenting the Gram-type determinant solution and Casorati-type determinant solution.
\end{abstract}

\section{Keywords}

Differential-Difference Davey-Stewartson System, Source Generalization Procedure, Discrete Gram-Type Determinant, Casorati-Type Determinant

\section{Introduction}

The study of discrete integrable system has become an active area of research for over thirty years. Various integrable discretization methods have been proposed to produce the discrete analogues of integrable systems. One powerful technique to find the integrable discretization is the Hirota's bilinear method [1]-[6]. The traditional Hirota's discretization of integrable equations relies on gauge invariance and soliton solutions, while the modified Hirota's approach [5] [6] emphasizes on discretizing integrable bilnear equations such that the resulting discrete bilinear equations have bilinear Bäcklund transformations.

The Davey-Stewartson system is an integrable $(2+1)$-dimensional generalization of the nonlinear Schrödinger system. In [7], the authors applied the modified Hirota's approach to the Davey-Stewartson system to produce an integrable differential-difference Davey-Stewartson system which is characterized by determinant solutions, bilinear Bäcklund transformation and lax pair. This differential-difference Davey-Stewartson system also can be derived as a reduction of a $(2+1)$-dimensional generalization of the Ablowitz-Ladik lattice [8].

Since the pioneering works of Mel'nikov [9], the soliton equations with self consistent sources have received considerable attention. Soliton equations with self consistent sources are integrable coupled generalization of the original soliton equations, and some of such type of equations have found important physical applications. A va- 
riety of methods have been proposed to deal with these soliton equations with sources, such as inverse scattering methods [9]-[13], Darboux transformation methods [14]-[17], Hirota's bilinear method and Wronskian technique [18]-[28] etc. However, most results have been achieved in continuous case. Comparatively less work has been done in discrete case. In view of this unsatisfactory situation, it would be interesting to produce new discrete soliton equations with self consistent sources.

In [27], a direct method, called the source generalization procedure, was proposed to construct and solve the soliton equations with self consistent sources. In this paper, we apply the source generalization procedure to construct and solve the differential-difference Davey-Stewartson system with self-consistent sources.

The outline of this paper is as follows. In Section 2, the differential-difference Davey-Stewartson system with self-consistent sources is produced and its Gram-type determinant solutions are presented. In Section 3, the Casorati-type determinant solutions to the differential-difference Davey-Stewartson system with self-consistent sources is derived. Finally, Section 4 is devoted to a conclusion.

\section{Constructing the Differential-Difference Davey-Stewartson System with Self-Consistent Sources}

In [7], a differential-difference Davey-Stewartson system which is an integrable discretization of the DSI system is proposed, and the double-Casorati and Grammian determinants solutions to this discrete Davey-Stewartson system are derived. In this section, we first review the Grammian determinant solutions for the discrete DaveyStewartson system and then apply the source generation procedure to this system to produce a differential-difference Davey-Stewartson system with self-consistent sources.

The differential-difference Davey-Stewartson system reads [7]

$$
\begin{gathered}
i v_{t}+\alpha_{1} \mathrm{e}^{u(n-1)+u(n+1)-2 u} v(n-1)+\alpha_{2} \mathrm{e}^{u(k-1)+u(k+1)-2 u} v(k+1)-\left(\alpha_{1}+\alpha_{2}\right) v=0, \\
-i w_{t}+\alpha_{1} \mathrm{e}^{u(n-1)+u(n+1)-2 u} w(n+1)+\alpha_{2} \mathrm{e}^{u(k-1)+u(k+1)-2 u} w(k-1)-\left(\alpha_{1}+\alpha_{2}\right) w=0, \\
z_{1}-z_{1} \mathrm{e}^{u(n+1, k+1)+u-u(k+1)-u(n+1)}+z_{2} v(k+1) w(n+1)=0,
\end{gathered}
$$

where $\alpha_{1}, \alpha_{2}, z_{1}$ and $z_{2}$ are constants. In Equations (1)-(3) and in the following we always use a notational simplification for $f(n, k, t)$ by writing explicitly a discrete variable only when it is shifted from its position. For example,

$$
f \equiv f(n, k, t), \quad f(n+1) \equiv f(n+1, k, t), \quad f(k-1) \equiv f(n, k-1, t), \quad f(n+1, k-1) \equiv f(n+1, k-1, t) .
$$

If we apply the dependent variables transformations

$$
u=\ln F, \quad v=\mathrm{e}^{-i\left(\alpha_{1}+\alpha_{2}\right) t} G / F, \quad w=\mathrm{e}^{i\left(\alpha_{1}+\alpha_{2}\right) t} H / F,
$$

Equations (1)-(3) can be transformed into the following bilinear Equations [7] [8]:

$$
\begin{gathered}
{\left[i D_{t}+\alpha_{1} \mathrm{e}^{-D_{n}}+\alpha_{2} \mathrm{e}^{D_{k}}\right] G \cdot F=0,} \\
{\left[i D_{t}+\alpha_{1} \mathrm{e}^{-D_{n}}+\alpha_{2} \mathrm{e}^{D_{k}}\right] F \cdot H=0,} \\
z_{1}\left[\mathrm{e}^{1 / 2\left(D_{n}-D_{k}\right)}-\mathrm{e}^{1 / 2\left(D_{n}+D_{k}\right)}\right] F \cdot F+z_{2} \mathrm{e}^{1 / 2\left(D_{n}-D_{k}\right)} G \cdot H=0,
\end{gathered}
$$

where, as usual, the bilinear operators $D_{t}$ and $\exp \left(\delta D_{n}\right)$ [28] are defined as:

$$
\begin{gathered}
\left.D_{t}^{m} a \cdot b \equiv\left(\frac{\partial}{\partial t}-\frac{\partial}{\partial t^{\prime}}\right)^{m} a(t) b\left(t^{\prime}\right)\right|_{t^{\prime}=t}, \\
\exp \left(\delta D_{n}\right) a \cdot b \equiv a(n+\delta) b(n-\delta) .
\end{gathered}
$$

The Grammian determinant solutions for the differential-difference Davey-Stewartson system (5)-(7) is given by [7]:

$$
F=|C+\Omega|=|\boldsymbol{F}|
$$




$$
G=\frac{z_{1}}{\alpha_{1}}\left|\begin{array}{cc}
\boldsymbol{F} & \Phi(n+1) \\
\Psi^{\prime}(-k+1)^{\mathrm{T}} & 0
\end{array}\right|, \quad H=\frac{1}{z_{2} \alpha_{2}}\left|\begin{array}{cc}
\boldsymbol{F} & \Psi(k+1) \\
\Phi^{\prime}(-n+1)^{\mathrm{T}} & 0
\end{array}\right|,
$$

where $\boldsymbol{F}$ is a $(M+N) \times(M+N)$ matrix, $C=\left(c_{\mu \nu}\right)$ is a $(M+N) \times(M+N)$ matrix of constant elements $c_{\mu v}(\mu, v=1,2, \cdots, M+N), \Omega$ is a $(M+N) \times(M+N)$ matrix with block structure, and $\Phi, \Phi^{\prime}, \Psi, \Psi^{\prime}$ are $M+N$ column vectors

$$
\begin{aligned}
\Omega= & \left(\begin{array}{cc}
\int_{-\infty}^{t} \phi_{r}(n) \phi_{j}^{\prime}(-n) \mathrm{d} t & \\
& \int_{t}^{+\infty} \psi_{s}(k) \psi_{l}^{\prime}(-k) \mathrm{d} t
\end{array}\right), \\
& \Phi(n)=\left(\phi_{1}(n), \cdots, \phi_{M}(n) ; 0, \cdots, 0\right)^{\mathrm{T}}, \\
& \Phi^{\prime}(-n)=\left(\phi_{1}^{\prime}(-n), \cdots, \phi_{M}^{\prime}(-n) ; 0, \cdots, 0\right)^{\mathrm{T}}, \\
& \Psi(k)=\left(0, \cdots, 0 ; \psi_{1}(k), \cdots, \phi_{N}(k)\right)^{\mathrm{T}}, \\
& \Psi^{\prime}(-k)=\left(0, \cdots, 0 ; \psi_{1}(-k), \cdots, \phi_{N}^{\prime}(-k)\right)^{\mathrm{T}},
\end{aligned}
$$

with $\phi_{r}(n, t), \phi_{j}^{\prime}(n, t), \psi_{s}(k, t), \psi_{l}^{\prime} k(n, t), \quad r, j \in\{1, \cdots, M\}, s, l \in\{1, \cdots, N\}$, satisfying the following equations:

$$
\begin{gathered}
i \frac{\partial \phi_{r}(n)}{\partial t}=-\alpha_{1} \phi_{r}(n-1), \quad i \frac{\partial \phi_{j}^{\prime}(-n)}{\partial t}=\alpha_{1} \phi_{j}(-n-1), \\
i \frac{\partial \psi_{s}(k)}{\partial t}=\alpha_{2} \psi_{s}(k-1), \quad i \frac{\partial \psi_{l}^{\prime}(-k)}{\partial t}=-\alpha_{2} \psi_{l}^{\prime}(-k-1) .
\end{gathered}
$$

We are now in a position to construct the differential-difference Davey-Stewartson system with self-consistent sources by applying the source generation procedure. Firstly, we change Grammian determinant solutions (8)(11) of Equations (5)-(7) to the following form:

$$
\begin{gathered}
F=|C(t)+\Omega|=|\boldsymbol{F}|, \\
G=\frac{z_{1}}{\alpha_{1}}\left|\begin{array}{cc}
\boldsymbol{F} & \Phi(n+1) \\
\Psi^{\prime}(-k+1)^{\mathrm{T}} & 0
\end{array}\right|, \quad H=\frac{1}{z_{2} \alpha_{2}}\left|\begin{array}{cc}
\boldsymbol{F} & \Psi(k+1) \\
\Phi^{\prime}(-n+1)^{\mathrm{T}} & 0
\end{array}\right|,
\end{gathered}
$$

where the $(M+N) \times(M+N)$ matrix $C(t)=\left(c_{\mu v}(t)\right)$ satisfies

$$
c_{\mu v}(t)= \begin{cases}c_{\mu}(t), & \mu=v \text { and } 1 \leq \mu \leq K \leq M+N, \\ c_{\mu}, & \text { otherwise, }\end{cases}
$$

with $c_{\mu}(t)$ being an arbitrary function of $t, K$ being a positive integer, and $\Omega, \Phi, \Phi^{\prime}, \Psi, \Psi^{\prime}$ are defined as before.

Using Equations (10)-(11), we can calculate the $t$-derivatives of the $F, G, H$ in (12)-(13) in following way:

$$
\begin{aligned}
& F_{t}=\sum_{r=1}^{K} \dot{c}_{r}(t)\left|A_{r r}\right|+\left|\begin{array}{cc}
\boldsymbol{F} & \Psi(k) \\
\Psi^{\prime}(-k)^{\mathrm{T}} & 0
\end{array}\right|-\left|\begin{array}{cc}
\boldsymbol{F} & \Phi(n) \\
\Phi^{\prime}(-n)^{\mathrm{T}} & 0
\end{array}\right|, \\
& i G_{t}=\frac{z_{1}}{\alpha_{1}}\left\{i \sum_{r=1}^{K} \dot{c}_{r}(t)\left|\begin{array}{cc}
A_{r r} & \Phi_{r}(n+1) \\
\Psi_{r}^{\prime}(-k+1)^{\mathrm{T}} & 0
\end{array}\right|+i\left|\begin{array}{ccc}
\boldsymbol{F} & \Phi(n+1) & \Psi(k) \\
\Psi^{\prime}(-k+1)^{\mathrm{T}} & 0 & 0 \\
\Psi^{\prime}(-k)^{\mathrm{T}} & 0 & 0
\end{array}\right|\right. \\
& \left.-i\left|\begin{array}{ccc}
\boldsymbol{F} & \Phi(n+1) & \Phi(n) \\
\Psi^{\prime}(-k+1)^{\mathrm{T}} & 0 & 0 \\
\Phi^{\prime}(-n)^{\mathrm{T}} & 0 & 0
\end{array}\right|-\alpha_{2}\left|\begin{array}{cc}
\boldsymbol{F} & \Phi(n+1) \\
\Psi^{\prime}(-k)^{\mathrm{T}} & 0
\end{array}\right|-\alpha_{1}\left|\begin{array}{cc}
\boldsymbol{F} & \Phi(n) \\
\Psi^{\prime}(-k+1)^{\mathrm{T}} & 0
\end{array}\right|\right\},
\end{aligned}
$$




$$
\begin{aligned}
i H_{t}= & \frac{1}{z_{2} \alpha_{2}}\left\{i \sum_{r=1}^{K} \dot{c}_{r}(t)\left|\begin{array}{cc}
A_{r r} & \Phi_{r}(n+1) \\
\Psi_{r}^{\prime}(-k+1)^{\mathrm{T}} & 0
\end{array}\right|+i\left|\begin{array}{ccc}
\boldsymbol{F} & \Psi(k+1) & \Psi(k) \\
\Phi^{\prime}(-n+1)^{\mathrm{T}} & 0 & 0 \\
\Psi^{\prime}(-k)^{\mathrm{T}} & 0 & 0
\end{array}\right|\right. \\
& \left.-i\left|\begin{array}{ccc}
\boldsymbol{F} & \Psi(k+1) & \Phi(n) \\
\Phi^{\prime}(-n+1)^{\mathrm{T}} & 0 & 0 \\
\Phi^{\prime}(-n)^{\mathrm{T}} & 0 & 0
\end{array}\right|+\alpha_{2}\left|\begin{array}{cc}
\boldsymbol{F} & \Psi(k) \\
\Phi^{\prime}(-n+1)^{\mathrm{T}} & 0
\end{array}\right|+\alpha_{1}\left|\begin{array}{cc}
\boldsymbol{F} & \Psi(k+1) \\
\Phi^{\prime}(-n)^{\mathrm{T}} & 0
\end{array}\right|\right\},
\end{aligned}
$$

where $A_{k l}$ denotes a matrix resulting from eliminating the $k$ th row and $l$ th column from the matrix $\boldsymbol{F}$, and $\Phi_{r}(n), \Psi_{r}^{\prime}(-k)$ denote vectors resulting from eliminating the $r$ th element from $\Phi(n), \Psi^{\prime}(-k)$ respectively.

Other functions appearing in Equations (5)-(7) such as $G(n-1), F(n+1), G(k+1), F(k-1), F(n-1)$, $H(n+1), F(k+1), H(k-1), G(k+1), F(n+1, k+1)$ can also be expressed in terms of Grammian determinants which are the same as the results given in [7].

Substituting Equations (15), (17) and $G(n-1), F(n+1), G(k+1), F(k-1)$ expressed by means of Grammian determinants given in [7] into the left side of Equation (6), and then applying the Jacobi identities for the determinants [28], we finally obtain

$$
i \frac{z_{1}}{\alpha_{1}} \sum_{r=1}^{K} \dot{c}_{r}(t)\left(\left|\begin{array}{cc}
A_{r r} & \Phi_{r}(n+1) \\
\Psi_{r}^{\prime}(-k+1)^{\mathrm{T}} & 0
\end{array}\right||F|-\left|\begin{array}{cc}
\boldsymbol{F} & \Phi(n+1) \\
\Psi^{\prime}(-k+1)^{\mathrm{T}} & 0
\end{array}\right|\left|A_{r r}\right|\right),
$$

Using the Jacobi identities for the determinants again, Equation (22) is equal to

$$
i \frac{z_{1}}{\alpha_{1}} \sum_{r=1}^{K} \dot{c}_{r}(t)\left|\begin{array}{c}
A_{r, 0} \\
\Psi^{\prime}(-k+1)^{\mathrm{T}}
\end{array}\right|\left|A_{0, r} \quad \Phi(n+1)\right|,
$$

where $A_{r, 0}, A_{0, j}$ denote matrices resulting from eliminating the $r$ th row and $j$ th column, respectively, from the matrix $\boldsymbol{F}$.

If we introduce two new fields $P_{r}, Q_{r}$ for $r=1,2, \cdots, K$ defined by

$$
P_{r}=\sqrt{\dot{c}_{r}(t)}\left|\begin{array}{c}
A_{r, 0} \\
\Psi^{\prime}(-k+1)^{\mathrm{T}}
\end{array}\right|, \quad Q_{r}=\sqrt{\dot{c}_{r}(t)}\left|A_{0, r} \quad \Phi(n+1)\right|,
$$

then we have shown that $F, G$ given in (12)-(13) and $P_{r}, Q_{r}, r=1,2, \cdots, K$ given in (20) satisfy the following bilinear equation:

$$
\left[i D_{t}+\alpha_{1} \mathrm{e}^{-D_{n}}+\alpha_{2} \mathrm{e}^{D_{k}}\right] G \cdot F=i \frac{Z_{1}}{\alpha_{1}} \sum_{r=1}^{K} P_{r} Q_{r} .
$$

In the same way, substituting (15) (17) and $H(n+1), F(n-1), H(k-1), G(k+1)$ expressed by means of Grammian determinants given in [7] into the left side of the Equation (6), and then applying the Jacobi identities for the determinants, we finally obtain

$$
i \frac{1}{z_{2} \alpha_{2}} \sum_{r=1}^{K} \dot{c}_{r}(t)\left(\left|\begin{array}{cc}
F & \Psi(k+1) \\
\Phi^{\prime}(-n+1)^{\mathrm{T}} & 0
\end{array}\right|\left|A_{r r}\right|-\left|\begin{array}{cc}
A_{r r} & \Phi_{r}(n+1) \\
\Psi_{r}^{\prime}(-k+1)^{\mathrm{T}} & 0
\end{array}\right||F|\right) .
$$

Using the Jacobi identities for the determinants again, Equation (22) is equal to

$$
-i \frac{1}{z_{2} \alpha_{2}} \sum_{r=1}^{K} \dot{c}_{r}(t)\left|\begin{array}{c}
A_{r, 0} \\
\Phi^{\prime}(-n+1)^{\mathrm{T}}
\end{array}\right|\left|A_{0, r} \quad \Psi(k+1)\right| .
$$

If we introduce another two new fields $J_{r}, L_{r}$ for $r=1,2, \cdots, K$ defined by

$$
J_{r}=\sqrt{\dot{c}_{r}(t)}\left|\begin{array}{c}
A_{r, 0} \\
\Phi^{\prime}(-n+1)^{\mathrm{T}}
\end{array}\right|, \quad L_{r}=\sqrt{\dot{c}_{r}(t)}\left|A_{0, r} \quad \Psi(k+1)\right|
$$


then we have shown that $F, H$ given in (12)-(13) and $J_{r}, L_{r}, r=1,2, \cdots, K$ given in (24) satisfy the following bilinear equation:

$$
\left[i D_{t}+\alpha_{1} \mathrm{e}^{-D_{n}}+\alpha_{2} \mathrm{e}^{D_{k}}\right] F \cdot H=-i \frac{1}{Z_{2} \alpha_{2}} \sum_{r=1}^{K} J_{r} L_{r} .
$$

There are more quadratic relations between the fields introduced. For example, the determinant identities

$$
-\left|\begin{array}{cc}
\boldsymbol{F} & \Phi(n+1) \\
\Phi^{\prime}(-n)^{\mathrm{T}} & 0
\end{array}\right|\left|\begin{array}{c}
A_{r, 0} \\
\Psi^{\prime}(-k+1)^{\mathrm{T}}
\end{array}\right|+|\boldsymbol{F}|\left|\begin{array}{cc}
A_{r, 0} & \Phi_{r}(n+1) \\
\Psi^{\prime}(-k+1)^{\mathrm{T}} & 0 \\
\Phi^{\prime}(-n)^{\mathrm{T}} & 0
\end{array}\right|+\left|\begin{array}{cc}
\boldsymbol{F} & \Phi(n+1) \\
\Psi^{\prime}(-k+1)^{\mathrm{T}} & 0
\end{array}\right| \begin{gathered}
A_{r, 0} \\
\Phi^{\prime}-n^{\mathrm{T}}
\end{gathered} \mid=0,
$$

and

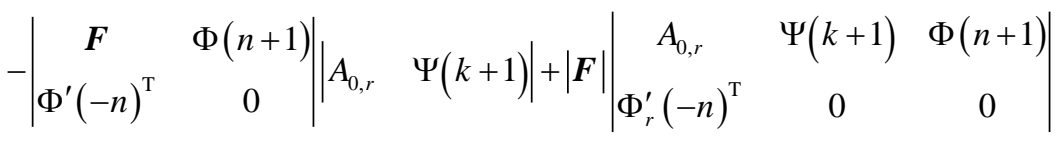

$$
\begin{aligned}
& +\left|\begin{array}{cc}
\boldsymbol{F} & \Psi(k+1) \\
\Phi^{\prime}(-n)^{\mathrm{T}} & 0
\end{array}\right|\left|A_{0, r} \quad \Phi(n+1)\right|=0,
\end{aligned}
$$

for $r=1,2, \cdots, K$ yield the bilinear equations

$$
z_{1}\left(\mathrm{e}^{1 / 2 D_{n}}-\mathrm{e}^{-1 / 2 D_{n}}\right) F \cdot P_{r}+i \mathrm{e}^{-1 / 2 D_{n}} G \cdot J_{r}=0,
$$

and

$$
\alpha_{1}\left(\mathrm{e}^{1 / 2 D_{n}}-\mathrm{e}^{-1 / 2 D_{n}}\right) F \cdot L_{r}+i \alpha_{2} Z_{2} \mathrm{e}^{1 / 2 D_{n}} H \cdot Q_{r}=0 .
$$

Similarly, bilinear equations

$$
z_{1} \alpha_{2}\left(\mathrm{e}^{-1 / 2 D_{k}}-\mathrm{e}^{1 / 2 D_{k}}\right) F \cdot Q_{r}-i \alpha_{1} \mathrm{e}^{1 / 2 D_{k}} G \cdot L_{r}=0,
$$

and

$$
\left(\mathrm{e}^{-1 / 2 D_{k}}-\mathrm{e}^{1 / 2 D_{k}}\right) F \cdot J_{r}-i z_{2} \mathrm{e}^{-1 / 2 D_{k}} H \cdot P_{r}=0,
$$

for $r=1,2, \cdots, K$ can be derived from the determinant identities

$$
\begin{aligned}
& -\left|\begin{array}{cc}
\boldsymbol{F} & \Psi(k) \\
\Psi^{\prime}(-k+1)^{\mathrm{T}} & 0
\end{array}\right|\left|A_{0, r} \quad \Phi(n+1)\right|+|\boldsymbol{F}|\left|\begin{array}{ccc}
A_{0, r} & \Phi(n+1) & \Psi(k) \\
\Psi^{\prime}(-k+1)^{\mathrm{T}} & 0 & 0
\end{array}\right| \\
& +\left|\begin{array}{cc}
\boldsymbol{F} & \Phi(n+1) \\
\Psi^{\prime}(-k+1)^{\mathrm{T}} & 0
\end{array}\right| A_{0, r} \quad \Psi(k) \mid=0,
\end{aligned}
$$

and

$$
-\left|\begin{array}{cc}
\boldsymbol{F} & \Psi(k) \\
\Psi^{\prime}(-k+1)^{\mathrm{T}} & 0
\end{array}\right|\left|\begin{array}{c}
A_{r, 0} \\
\Phi^{\prime}(-n+1)^{\mathrm{T}}
\end{array}\right|+|\boldsymbol{F}|\left|\begin{array}{cc}
A_{r, 0} & \Psi_{r}(k) \\
\Phi^{\prime}(-n+1)^{\mathrm{T}} & 0 \\
\Psi^{\prime}(-k+1)^{\mathrm{T}} & 0
\end{array}\right|+\left|\begin{array}{cc}
\boldsymbol{F} & \Psi(k) \\
\mid \Phi^{\prime}(-n+1)^{\mathrm{T}} & 0
\end{array}\right| \begin{gathered}
A_{r, 0} \\
\Psi^{\prime}(-k+1)^{\mathrm{T}}
\end{gathered} \mid=0 .
$$

The determinant identities (26)-(27) and (32)-(33) are special cases of the pfaffian identity [28],

$$
\begin{aligned}
& \left(a_{1}, a_{2}, \cdots, a_{N-1}, \alpha, \beta, \gamma\right)\left(a_{1}, a_{2}, \cdots, a_{N-1}, \delta\right)-\left(a_{1}, a_{2}, \cdots, a_{N-1}, \alpha, \beta, \delta\right)\left(a_{1}, a_{2}, \cdots, a_{N-1}, \gamma\right) \\
& \quad+\left(a_{1}, a_{2}, \cdots, a_{N-1}, \alpha, \gamma, \delta\right)\left(a_{1}, a_{2}, \cdots, a_{N-1}, \beta\right)-\left(a_{1}, a_{2}, \cdots, a_{N-1}, \beta, \gamma, \delta\right)\left(a_{1}, a_{2}, \cdots, a_{N-1}, \alpha\right)=0 .
\end{aligned}
$$

So bilinear Equations (7), (21), (25) and (28)-(31) for $r=1,2, \cdots, K$ construct the differential-difference Da- 
vey-Stewartson system with self-consistent sources, and functions $F, G, H$ and $P_{r}, Q_{r}, J_{r}, L_{r}$ for $r=1,2, \cdots, K$ in Equations (3), (12), (20), (24) are the Gram-type determinant solutions of the differential-difference DaveyStewartson system with self-consistent sources. Under the dependent variable transformations

$$
u=\ln F, \quad v=\frac{G}{F}, \quad w=\frac{H}{F}, \quad \tilde{P}_{r}=\frac{P_{r}}{F}, \quad \tilde{Q}_{r}=\frac{Q_{r}}{F}, \quad \tilde{L}_{r}=\frac{L_{r}}{F}, \quad \tilde{J}_{r}=\frac{J_{r}}{F},
$$

the bilinear Equations (7), (21), (25) and (28)-(31) for $r=1,2, \cdots, K$ are transformed into the following nonlinear equations:

$$
\begin{gathered}
z_{1}-z_{1} \mathrm{e}^{u(n+1, k+1)+u-u(n+1)-u(k+1)}+z_{2} v(k+1) w(n+1)=0, \\
i v_{t}+\alpha_{1} v(n-1) \mathrm{e}^{u(n-1)+u(n+1)-2 u}+\alpha_{2} v(k+1) \mathrm{e}^{u(k-1)+u(k+1)-2 u}=i \frac{z_{1}}{\alpha_{1}} \sum_{r=1}^{K} \tilde{P}_{r} \tilde{Q}_{r}, \\
i w_{t}-\alpha_{1} w(n+1) \mathrm{e}^{u(n-1)+u(n+1)-2 u}-\alpha_{2} w(k-1) \mathrm{e}^{u(k-1)+u(k+1)-2 u}=i \frac{1}{z_{2} \alpha_{2}} \sum_{r=1}^{K} \tilde{J}_{r} \tilde{L}_{r}, \\
i v \tilde{J}_{r}(n+1)+z_{1}\left(\tilde{P}_{r}-\tilde{P}_{r}(n+1)\right)=0, \\
\alpha_{1}\left(\tilde{L}_{r}-\tilde{L}_{r}(n+1)\right)+i \alpha_{2} z_{2} \tilde{Q}_{r} w(n+1)=0, \\
z_{1} \alpha_{2}\left(\tilde{Q}_{r}-\tilde{Q}_{r}(k-1)\right)-i \alpha_{1} v \tilde{L}_{r}(k-1)=0, \\
i z_{2} \tilde{P}_{r} w(k-1)+\tilde{J}_{r}(k-1)-\tilde{J}_{r}=0 .
\end{gathered}
$$

\section{Casorati-Type Determinant Solutions of the Differential-Difference Davey-Stewartson System with Self-Consistent Sources}

It is shown in [7] that the differential-difference Davey-Stewartson system exhibits N-soliton solutions expressed by means of two types of determinants, double-Casorati and Grammian determinants. It is natural to consider if the differential-difference Davey-Stewartson system with self-consistent sources have two types of determinant solutions. In this section, we shall derive another class of determinant solutions, Casorati-type determinant solutions to the differential-difference Davey-Stewartson system with self-consistent sources (7), (21), (25) and (28)-(31) for $r=1,2, \cdots, K$.

Let us introduce the following double-Casorati determinant:

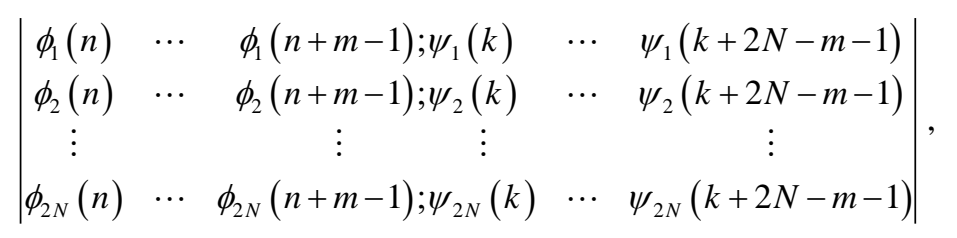

where for $r=1,2, \cdots, 2 N$,

$$
\begin{aligned}
& \phi_{r}(n, t)=\phi_{r 1}(n, t)+i(-1)^{r} C_{r}(t) \phi_{r 2}(n, t), \\
& \psi_{r}(k, t)=\psi_{r 1}(k, t)+i(-1)^{r} C_{r}(t) \psi_{r 2}(k, t),
\end{aligned}
$$

in which $C_{r}(t)$ satisfies

$$
C_{r}(t)= \begin{cases}c_{r}(t), & 1 \leq r \leq K \leq 2 N \\ c_{r}, & \text { otherwise }\end{cases}
$$

with $c_{r}(t)$ being an arbitrary function of $t, c_{r}$ is an arbitrary constant and $K$ being a positive integer, and $\phi_{r 1}(n, t), \phi_{r 2}(n, t), \psi_{r 1}(k, t), \psi_{r 2}(n, t)$ satisfy the following equations:

$$
i \frac{\partial}{\partial t} \phi_{r 1}(n)=-\alpha_{1} \phi_{r 1}(n-1), \quad i \frac{\partial}{\partial t} \phi_{r 2}(n)=-\alpha_{1} \phi_{r 2}(n-1),
$$




$$
i \frac{\partial}{\partial t} \psi_{r 1}(k)=\alpha_{2} \psi_{r 1}(k-1), \quad i \frac{\partial}{\partial t} \psi_{r 2}(k)=\alpha_{2} \psi_{r 2}(k-1) .
$$

From now on the determinant (42) will, for simplicity, be denoted as

$$
\left|0,1, \cdots, m-1 ; 0^{\prime}, 1^{\prime}, \cdots,(2 N-m-1)^{\prime}\right| .
$$

Taking into account Equations (42)-(48), we can state the following Proposition:

Proposition 1 The solutions to Equations (7) (21) (25) and (28)-(31) for $r=1,2, \cdots, K$ can be expressed as the following double-Casorati type determinants:

$$
\begin{gathered}
F=\left|0,1, \cdots, m-1 ; 0^{\prime}, 1^{\prime}, \cdots,(2 N-m-1)^{\prime}\right|, \\
G=z_{1}\left|0,1, \cdots, m ; 0^{\prime}, 1^{\prime}, \cdots,(2 N-m-2)^{\prime}\right|, \\
H=\frac{1}{z_{2}}\left|0,1, \cdots, m-2 ; 0^{\prime}, 1^{\prime}, \cdots,(2 N-m)^{\prime}\right|, \\
P_{r}=i \sqrt{\dot{c}_{r}(t)}\left(d_{0}, \cdots, d_{m-1}, d_{0}^{*}, \cdots, d_{2 N-m-2}^{*}, 2 N, \cdots, \hat{r}, \cdots, 1\right), \\
Q_{r}=\alpha_{1} \sqrt{\dot{c}_{r}(t)}\left(d_{0}, \cdots, d_{m}, d_{0}^{*}, \cdots, d_{2 N-m-1}^{*}, 2 N, \cdots, 1, \alpha_{r}\right), \\
J_{r}=\sqrt{\dot{c}_{r}(t)}\left(d_{0}, \cdots, d_{m-2}, d_{0}^{*}, \cdots, d_{2 N-m-1}^{*}, 2 N, \cdots, \hat{r}, \cdots, 1\right), \\
L_{r}=-i \alpha_{2} \sqrt{\dot{c}_{r}(t)}\left(d_{0}, \cdots, d_{m-1}, d_{0}^{*}, \cdots, d_{2 N-m}^{*}, 2 N, \cdots, 1, \alpha_{r}\right),
\end{gathered}
$$

where the pfaffian elements are defined by

$$
\begin{gathered}
\left(d_{l}, j\right)=\phi_{j}(n+l), \quad\left(d_{s}^{*}, j\right)=\psi_{j}(k+s), \\
(i, j)=0, \quad\left(d_{l}, d_{s}^{*}\right)=0, \quad\left(d_{l}, d_{s}\right)=0, \quad\left(d_{l}^{*}, d_{s}^{*}\right)=0, \\
\left(d_{l}, \alpha_{r}\right)=\phi_{r 2}(n+l), \quad\left(d_{s}^{*}, \alpha_{r}\right)=\phi_{r 2}(k+s),
\end{gathered}
$$

in which $l, s$ are integers, $i, j=1,2, \cdots, 2 N, r=1,2, \cdots, K$, and ${ }^{\wedge}$ in the pfaffians indicates deletion of the letter under it.

Proof: The double Casorati determinants in (11)-(13) can be expressed by pfaffians [28] in the following way:

$$
\begin{gathered}
F=\left(d_{0}, \cdots, d_{m-1}, d_{0}^{*}, \cdots, d_{2 N-m-1}^{*}, 2 N, \cdots, 1\right), \\
G=z_{1}\left(d_{0}, \cdots, d_{m}, d_{0}^{*}, \cdots, d_{2 N-m-2}^{*}, 2 N, \cdots, 1\right), \\
H=\frac{1}{z_{2}}\left(d_{0}, \cdots, d_{m-2}, d_{0}^{*}, \cdots, d_{2 N-m}^{*}, 2 N, \cdots, 1\right),
\end{gathered}
$$

where the pfaffian elements are given in (56)-(58).

We first show that functions (49)-(55) satisfy Equations (21) and (25). Using Equations (43)-(47), we can calculate the following differential and difference formula for $F, G, H$ :

$$
\begin{gathered}
i F_{t}=-\alpha_{1}\left(d_{-1}, d_{1}, \cdots, d_{m-1}, d_{0}^{*}, \cdots, d_{2 N-m-1}^{*}, 2 N, \cdots, 1\right)+\alpha_{2}\left(d_{0}, \cdots, d_{m-1}, d_{-1}^{*}, d_{1}^{*}, \cdots, d_{2 N-m-1}^{*}, 2 N, \cdots, 1\right) \\
+\sum_{r=1}^{K} \dot{c}_{r}(t)\left(d_{0}, \cdots, d_{m-1}, d_{0}^{*}, \cdots, d_{2 N-m-1}^{*}, 2 N, \cdots, \hat{r}, \cdots, 1, \alpha_{r}\right), \\
F_{n+1}=\left(d_{1}, \cdots, d_{m}, d_{0}^{*}, \cdots, d_{2 N-m-1}^{*}, 2 N, \cdots, 1\right), \\
F_{k-1}=\left(d_{0}, \cdots, d_{m-1}, d_{-1}^{*}, d_{0}^{*}, \cdots, d_{2 N-m-2}^{*}, 2 N, \cdots, 1\right),
\end{gathered}
$$




$$
\begin{gathered}
i G_{t}=z_{1}\left[-\alpha_{1}\left(d_{-1}, d_{1}, \cdots, d_{m}, d_{0}^{*}, \cdots, d_{2 N-m-2}^{*}, 2 N, \cdots, 1\right)+\alpha_{2}\left(d_{0}, \cdots, d_{m}, d_{-1}^{*}, d_{1}^{*}, \cdots, d_{2 N-m-2}^{*}, 2 N, \cdots, 1\right)\right. \\
\left.+\sum_{r=1}^{K} \dot{c}_{r}(t)\left(d_{0}, \cdots, d_{m}, d_{0}^{*}, \cdots, d_{2 N-m-2}^{*}, 2 N, \cdots, \hat{r}, \cdots, 1, \alpha_{r}\right)\right], \\
G_{n-1}=z_{1}\left(d_{-1}, d_{0}, \cdots, d_{m-1}, d_{0}^{*}, \cdots, d_{2 N-m-2}^{*}, 2 N, \cdots, 1\right), \\
G_{k+1}=z_{1}\left(d_{0}, \cdots, d_{m}, d_{1}^{*}, \cdots, d_{2 N-m-1}^{*}, 2 N, \cdots, 1\right), \\
i H_{t}=\frac{1}{z_{2}}\left[-\alpha_{1}\left(d_{-1}, d_{1}, \cdots, d_{m-2}, d_{0}^{*}, \cdots, d_{2 N-m}^{*}, 2 N, \cdots, 1\right)+\alpha_{2}\left(d_{0}, \cdots, d_{m-2}, d_{-1}^{*}, d_{1}^{*}, \cdots, d_{2 N-m}^{*}, 2 N, \cdots, 1\right)\right. \\
\left.+\sum_{r=1}^{K} \dot{c}_{r}(t)\left(d_{0}, \cdots, d_{m-2}, d_{0}^{*}, \cdots, d_{2 N-m}^{*}, 2 N, \cdots, \hat{r}, \cdots, 1, \alpha_{r}\right)\right], \\
F_{n-1}=\left(d_{-1}, d_{0}, \cdots, d_{m-2}, d_{0}^{*}, \cdots, d_{2 N-m-1}^{*}, 2 N, \cdots, 1\right), \\
F_{k+1}=\left(d_{0}, \cdots, d_{m-1}, d_{1}^{*}, \cdots, d_{2 N-m}^{*}, 2 N, \cdots, 1\right), \\
H_{n+1}=\frac{1}{z_{2}}\left(d_{1}, \cdots, d_{m-1}, d_{0}^{*}, \cdots, d_{2 N-m}^{*}, 2 N, \cdots, 1\right), \\
H_{k-1}=\frac{1}{z_{2}}\left(d_{0}, \cdots, d_{m-2}, d_{-1}^{*}, d_{0}^{*}, \cdots, d_{2 N-m-1}^{*}, 2 N, \cdots, 1\right) .
\end{gathered}
$$

Substitution of Equations (52)-(55) and (62)-(72) into Equations (21) and (25) yields the following determinant identities, respectively:

$$
\begin{aligned}
& \left(d_{0}, \cdots, d_{m}, d_{0}^{*}, \cdots, d_{2 N-m-2}^{*}, 2 N, \cdots, \hat{r}, \cdots, 1, \alpha_{r}\right)\left(d_{0}, \cdots, d_{m-1}, d_{0}^{*}, \cdots, d_{2 N-m-1}^{*}, 2 N, \cdots, 1\right) \\
& \quad-\left(d_{0}, \cdots, d_{m}, d_{0}^{*}, \cdots, d_{2 N-m-2}^{*}, 2 N, \cdots, 1\right)\left(d_{0}, \cdots, d_{m-1}, d_{0}^{*}, \cdots, d_{2 N-m-1}^{*}, 2 N, \cdots, \hat{r}, \cdots, 1, \alpha_{r}\right) \\
& \quad-\left(d_{0}, \cdots, d_{m-1}, d_{0}^{*}, \cdots, d_{2 N-m-2}^{*}, 2 N, \cdots, \hat{r}, \cdots, 1\right)\left(d_{0}, \cdots, d_{m}, d_{0}^{*}, \cdots, d_{2 N-m-1}^{*}, 2 N, \cdots, 1, \alpha_{r}\right)=0,
\end{aligned}
$$

and

$$
\begin{aligned}
& \left(d_{0}, \cdots, d_{m-1}, d_{0}^{*}, \cdots, d_{2 N-m-1}^{*}, 2 N, \cdots, \hat{r}, \cdots, 1, \alpha_{r}\right)\left(d_{0}, \cdots, d_{m-2}, d_{0}^{*}, \cdots, d_{2 N-m}^{*}, 2 N, \cdots, 1\right) \\
& \quad-\left(d_{0}, \cdots, d_{m-1}, d_{0}^{*}, \cdots, d_{2 N-m-1}^{*}, 2 N, \cdots, 1\right)\left(d_{0}, \cdots, d_{m-2}, d_{0}^{*}, \cdots, d_{2 N-m}^{*}, 2 N, \cdots, \hat{r}, \cdots, 1, \alpha_{r}\right) \\
& \quad-\left(d_{0}, \cdots, d_{m-2}, d_{0}^{*}, \cdots, d_{2 N-m-1}^{*}, 2 N, \cdots, \hat{r}, \cdots, 1\right)\left(d_{0}, \cdots, d_{m-1}, d_{0}^{*}, \cdots, d_{2 N-m}^{*}, 2 N, \cdots, 1, \alpha_{r}\right)=0 .
\end{aligned}
$$

It is easy to show that (49)-(51) satisfy Equation (7). Now we prove that functions (49)-(55) satisfy Equations (28)-(31). From Equations (52)-(58), we can derive the difference formula for pfaffians $P_{r}, Q_{r}, J_{r}, L_{r}$, $r=1, \cdots, K$ as follows:

$$
\begin{gathered}
P_{r}(n+1)=i \sqrt{\dot{c}_{r}(t)}\left(d_{1}, \cdots, d_{m}, d_{0}^{*}, \cdots, d_{2 N-m-2}^{*}, 2 N, \cdots, \hat{j}, \cdots, 1\right), \\
J_{r}(n+1)=\sqrt{\dot{c}_{r}(t)}\left(d_{1}, \cdots, d_{m-1}, d_{0}^{*}, \cdots, d_{2 N-m-1}^{*}, 2 N, \cdots, \hat{j}, \cdots, 1\right), \\
L_{r}(n+1)=-i \alpha_{2} \sqrt{\dot{c}_{r}(t)}\left(d_{1}, \cdots, d_{m}, d_{0}^{*}, \cdots, d_{2 N-m}^{*}, 2 N, \cdots, 1, \beta_{r}\right), \\
Q_{r}(k-1)=\alpha_{1} \sqrt{\dot{c}_{r}(t)}\left(d_{0}, \cdots, d_{m}, d_{-1}^{*}, d_{0}^{*}, \cdots, d_{2 N-m-2}^{*}, 2 N, \cdots, 1, \beta_{r}\right), \\
L_{r}(k-1)=-i \alpha_{2} \sqrt{\dot{c}_{r}(t)}\left(d_{0}, \cdots, d_{m-1}, d_{-1}^{*}, d_{0}^{*}, \cdots, d_{2 N-m-1}^{*}, 2 N, \cdots, 1, \beta_{r}\right), \\
J_{r}(k-1)=\sqrt{\dot{c}_{r}(t)}\left(d_{0}, \cdots, d_{m-2}, d_{-1}^{*}, d_{0}^{*}, \cdots, d_{2 N-m-2}^{*}, 2 N, \cdots, \hat{j}, \cdots, 1\right) .
\end{gathered}
$$


Substituting Equations (59)-(60), (63)-(64), (71)-(72) and (75)-(80) into Equations (28)-(31), we obtain the following determinant identities, respectively:

$$
\begin{aligned}
& \left(d_{0}, \cdots, d_{m}, d_{0}^{*}, \cdots, d_{2 N-m-2}^{*}, 2 N, \cdots, \hat{r}, \cdots, 1, \alpha_{r}\right)\left(d_{0}, \cdots, d_{m-1}, d_{0}^{*}, \cdots, d_{2 N-m-1}^{*}, 2 N, \cdots, 1\right) \\
& -\left(d_{0}, \cdots, d_{m}, d_{0}^{*}, \cdots, d_{2 N-m-2}^{*}, 2 N, \cdots, 1\right)\left(d_{0}, \cdots, d_{m-1}, d_{0}^{*}, \cdots, d_{2 N-m-1}^{*}, 2 N, \cdots, \hat{r}, \cdots, 1, \alpha_{r}\right) \\
& -\left(d_{0}, \cdots, d_{m-1}, d_{0}^{*}, \cdots, d_{2 N-m-2}^{*}, 2 N, \cdots, \hat{r}, \cdots, 1\right)\left(d_{0}, \cdots, d_{m}, d_{0}^{*}, \cdots, d_{2 N-m-1}^{*}, 2 N, \cdots, 1, \alpha_{r}\right)=0, \\
& \left(d_{0}, \cdots, d_{m}, d_{0}^{*}, \cdots, d_{2 N-m-2}^{*}, 2 N, \cdots, \hat{r}, \cdots, 1, \alpha_{r}\right)\left(d_{0}, \cdots, d_{m-1}, d_{0}^{*}, \cdots, d_{2 N-m-1}^{*}, 2 N, \cdots, 1\right) \\
& \quad-\left(d_{0}, \cdots, d_{m}, d_{0}^{*}, \cdots, d_{2 N-m-2}^{*}, 2 N, \cdots, 1\right)\left(d_{0}, \cdots, d_{m-1}, d_{0}^{*}, \cdots, d_{2 N-m-1}^{*}, 2 N, \cdots, \hat{r}, \cdots, 1, \alpha_{r}\right) \\
& -\left(d_{0}, \cdots, d_{m-1}, d_{0}^{*}, \cdots, d_{2 N-m-2}^{*}, 2 N, \cdots, \hat{r}, \cdots, 1\right)\left(d_{0}, \cdots, d_{m}, d_{0}^{*}, \cdots, d_{2 N-m-1}^{*}, 2 N, \cdots, 1, \alpha_{r}\right)=0, \\
& \left(d_{0}, \cdots, d_{m}, d_{0}^{*}, \cdots, d_{2 N-m-2}^{*}, 2 N, \cdots, \hat{r}, \cdots, 1, \alpha_{r}\right)\left(d_{0}, \cdots, d_{m-1}, d_{0}^{*}, \cdots, d_{2 N-m-1}^{*}, 2 N, \cdots, 1\right) \\
& -\left(d_{0}, \cdots, d_{m}, d_{0}^{*}, \cdots, d_{2 N-m-2}^{*}, 2 N, \cdots, 1\right)\left(d_{0}, \cdots, d_{m-1}, d_{0}^{*}, \cdots, d_{2 N-m-1}^{*}, 2 N, \cdots, \hat{r}, \cdots, 1, \alpha_{r}\right) \\
& -\left(d_{0}, \cdots, d_{m-1}, d_{0}^{*}, \cdots, d_{2 N-m-2}^{*}, 2 N, \cdots, \hat{r}, \cdots, 1\right)\left(d_{0}, \cdots, d_{m}, d_{0}^{*}, \cdots, d_{2 N-m-1}^{*}, 2 N, \cdots, 1, \alpha_{r}\right)=0, \\
& \left(d_{0}, \cdots, d_{m}, d_{0}^{*}, \cdots, d_{2 N-m-2}^{*}, 2 N, \cdots, \hat{r}, \cdots, 1, \alpha_{r}\right)\left(d_{0}, \cdots, d_{m-1}, d_{0}^{*}, \cdots, d_{2 N-m-1}^{*}, 2 N, \cdots, 1\right) \\
& -\left(d_{0}, \cdots, d_{m}, d_{0}^{*}, \cdots, d_{2 N-m-2}^{*}, 2 N, \cdots, 1\right)\left(d_{0}, \cdots, d_{m-1}, d_{0}^{*}, \cdots, d_{2 N-m-1}^{*}, 2 N, \cdots, \hat{r}, \cdots, 1, \alpha_{r}\right) \\
& -\left(d_{0}, \cdots, d_{m-1}, d_{0}^{*}, \cdots, d_{2 N-m-2}^{*}, 2 N, \cdots, \hat{r}, \cdots, 1\right)\left(d_{0}, \cdots, d_{m}, d_{0}^{*}, \cdots, d_{2 N-m-1}^{*}, 2 N, \cdots, 1, \alpha_{r}\right)=0 .
\end{aligned}
$$

\section{Conclusions}

In this paper, we apply the source generation procedure to the differential-difference Davey-Stewartson system (1)-(3) to generate a differential-difference Davey-Stewartson system with self-consistent sources (35)-(41), and clarify the algebraic structures of the resulting coupled discrete system by expressing the solutions in terms of two types of determinants, Casorati-type determinant and Gram-type determinant.

In [29], a Davey-Stewartson equation with self-consistent sources is constructed. It would be of interest to find the proper reduction and certain continuous limits which give the Davey-Stewartson equation with selfconsistent sources investigated in [29] from the differential-difference Davey-Stewartson system with self-consistent sources (35)-(41).

\section{Acknowledgements}

The author would like to express her sincere thanks to Prof. Xing-Biao Hu for his helpful discussions and encouragement. This work was supported by the program of higher-level talents of Inner Mongolia University (2011153) and the National Natural Science Foundation of China (Grant No. 11102212).

\section{References}

[1] Hirota, R. (1977) Nonlinear Partial Difference Equations: I. A Difference Analogue of the Korteweg-de Vries Equation. Journal of the Physical Society of Japan, 43, 1424-1433. http://dx.doi.org/10.1143/JPSJ.43.1424

[2] Hirota, R. (1977) Nonlinear Partial Difference Equations: II. Discrete-Time Toda Equation. Journal of the Physical Society of Japan, 43, 2074-2078. http://dx.doi.org/10.1143/JPSJ.43.2074

[3] Hirota, R. (1977) Nonlinear Partial Difference Equations: III. Discrete Sine-Gordon Equation. Journal of the Physical Society of Japan, 43, 2079-2086. http://dx.doi.org/10.1143/JPSJ.43.2079

[4] Feng, B.F., Inoguchi, J., Kajiwara, K., Maruno, K. and Ohta, Y. (2013) Integrable Discretizations of the Dym Equation. Frontiers of Mathematics in China, 8, 1017-1029. http://dx.doi.org/10.1007/s11464-013-0321-y

[5] Hu, X.B. and Yu, G.F. (2007) Integrable Discretizations of the (2+1)-Dimensional Sinh-Gordon Equation. Journal of Physics A: Mathematical and Theoretical, 40, 12645-12659. http://dx.doi.org/10.1088/1751-8113/40/42/S10 
[6] Hu, X.B. and Yu, G.F. (2009) Integrable Semi-Discretizations and Full-Discretization of the Two-Dimensional Leznov Lattice. Journal of Difference Equations and Applications, 15, 233-252. http://dx.doi.org/10.1080/10236190802101695

[7] Gegenhasi, Hu, X.B. and Levi, D. (2006) On a Discrete Davey-Stewartson System. Inverse Problems, 22, 1677-1688. http://dx.doi.org/10.1088/0266-5611/22/5/009

[8] Tsuchida, T. and Dimakis, A. (2011) On a (2 + 1)-Dimensional Generalization of the Ablowitz-Ladik Lattice and Adiscrete Davey-Stewartson System. Journal of Physics A: Mathematical and Theoretical, 44, Article ID: 325206. http://dx.doi.org/10.1088/1751-8113/44/32/325206

[9] Mel'nikov, V.K. (1983) On Equations for Wave Interactions. Letters in Mathematical Physics, 7, 129-136. http://dx.doi.org/10.1007/BF00419931

[10] Mel'nikov, V.K. (1990) Integration of the Korteweg-de Vries Equation with a Source. Inverse Problems, 6, $233-246$. http://dx.doi.org/10.1088/0266-5611/6/2/007

[11] Mel'nikov, V.K. (1992) Integration of the Nonlinear Schrödinger Equation with a Source. Inverse Problems, 8, 133147. http://dx.doi.org/10.1088/0266-5611/8/1/009

[12] Zeng, Y.B., Ma, W.X. and Lin, R.L. (2000) Integration of the Soliton Hierarchy with Self-Consistent Sources. Journal of Mathematical Physics, 41, 5453-5489. http://dx.doi.org/10.1063/1.533420

[13] Lin, R.L., Zeng, Y.B. and Ma, W.X. (2001) Solving the KdV Hierarchy with Self-Consistent Sources by Inverse Scattering Method. Physica A: Statistical Mechanics and Its Applications, 291, 287-298. http://dx.doi.org/10.1016/S0378-4371(00)00519-7

[14] Zeng, Y.B., Ma, W.X. and Shao, Y.J. (2001) Two Binary Darboux Transformations for the KdV Hierarchy with SelfConsistent Sources. Journal of Mathematical Physics, 42, 2113-2128. http://dx.doi.org/10.1063/1.1357826

[15] Zeng, Y.B., Shao, Y.J. and Ma, W.X. (2002) Integral-Type Darboux Transformation for the mKdV Hierarchy with Self-Consistent Sources. Communications in Theoretical Physics, 38, 641-648. http://dx.doi.org/10.1088/0253-6102/38/6/641

[16] Xiao, T. and Zeng, Y.B. (2004) Generalized Darboux Transformations for the KP Equation with Self-Consistent Sources. Journal of Physics A: Mathematical and General, 37, 7143-7162. http://dx.doi.org/10.1088/0305-4470/37/28/006

[17] Liu, X.J. and Zeng, Y.B. (2005) On the Toda Lattice Equation with Self-Consistent Sources. Journal of Physics A: Mathematical and General, 38, 8951-8965. http://dx.doi.org/10.1088/0305-4470/38/41/008

[18] Hase, Y., Hirota, R., Ohta, Y. and Satsuma, J. (1989) Soliton Solutions of the Mel'nikov Equations. Journal of the Physical Society of Japan, 58, 2713-2720.

[19] Matsuno, Y. (1991) Bilinear Bäcklund Transformation for the KdV Equation with a Source. Journal of Physics A: Mathematical and General, 24, L273. http://dx.doi.org/10.1088/0305-4470/24/6/005

[20] Hu, X.B. (1991) Nonlinear Superposition Formula of the KdV Equation with a Source. Journal of Physics A: Mathematical and General, 24, 5489-5497. http://dx.doi.org/10.1088/0305-4470/24/23/015

[21] Matsuno, Y. (1990) KP Equation with a Source and Its Soliton Solutions. Journal of Physics A: Mathematical and General, 23, L1235. http://dx.doi.org/10.1088/0305-4470/23/23/009

[22] Zhang, D.J. (2002) The $N$-Soliton Solutions for the Modified KdV Equation with Self-Consistent Sources. Journal of the Physical Society of Japan, 71, 2649-2656. http://dx.doi.org/10.1143/JPSJ.71.2649

[23] Deng, S.F., Chen, D.Y. and Zhang, D.J. (2003) The Multisoliton Solutions of the KP Equation with Self-Consistent Sources. Journal of the Physical Society of Japan, 72, 2184-2192. http://dx.doi.org/10.1143/JPSJ.72.2184

[24] Zhang, D.J. and Chen, D.Y. (2003) The $N$-Soliton Solutions of the Sine-Gordon Equation with Self-Consistent Sources. Physica A: Statistical Mechanics and Its Applications, 321, 467-481. http://dx.doi.org/10.1016/S0378-4371(02)01742-9

[25] Zhang, D.J. (2003) The $N$-Soliton Solutions of Some Soliton Equations with Self-Consistent Sources. Chaos, Solitons and Fractals, 18, 31-43. http://dx.doi.org/10.1016/S0960-0779(02)00636-7

[26] Gegenhasi and Hu, X.-B. (2006) On an Integrable Differential-Difference Equation with a Source. Journal of Nonlinear Mathematical Physics, 13, 183-192. http://dx.doi.org/10.2991/jnmp.2006.13.2.3

[27] Hu, X.B. and Wang, H.Y. (2006) Construction of dKP and BKP Equation with Self-Consistent Sources. Inverse Problems, 22, 1903-1920. http://dx.doi.org/10.1088/0266-5611/22/5/022

[28] Hirota, R. (2004) Direct Method in Soliton Theory. Cambridge University Press, Cambridge. Edited and Translated by Nagai, A., Nimmo, J. and Gilson, C. (In English)

[29] Hu, J., Wang, H.Y. and Tam, H.W. (2008) Source Generation of the Davey-Stewartson Equation. Journal of Mathematical Physics, 49, Article ID: 013506. http://dx.doi.org/10.1063/1.2830432 